\title{
Representaciones Sociales y Formación Inicial Docente: El desafío de educar en ciudadanía
}

\author{
Social Representations and teacher education: the challenge for educating in \\ citizenship
}

\author{
Héctor Gómez Cuevas \\ University of British Columbia, Canadá. \\ Contacto: hrgomez@uc.cl \\ Felipe Pizarro Cerda \\ Universidad Católica Silva Henríquez, Chile. \\ Contacto: fpizarro@ucsh.cl
}

\begin{abstract}
Resumen
El presente artículo se propone develar las representaciones sociales de estudiantes de Pedagogía en Historia, Geografía y Ciencias Sociales de dos universidades de la Región Metropolitana en torno a la ciudadanía y la formación ciudadana, y su vinculación con el currículo de Formación Inicial Docente. En este sentido, el estudio adhiere a un paradigma constructivista del campo de la investigación en base a un enfoque cualitativo. El diseño se vincula con un estudio de casos y los datos fueron recabados a partir de las técnicas de análisis documental y grupo focal para posteriormente ser analizados mediante un análisis de contenido. Los resultados del estudio demostraron que existen representaciones sociales de los estudiantes ligados a diferentes paradigmas de la ciudadanía como a diferentes tipos de formación ciudadana. Además, se señala que las representaciones sociales de los estudiantes poseen una mezcla de elementos teóricos respecto a la ciudadanía y también a la formación ciudadana, donde se observan en una misma representación social características de un paradigma comunitarista y de uno liberal, o de un tipo de formación ciudadana política y de una social.
\end{abstract}

Palabras Clave: Currículum, Formación Docente, Ciudadanía, Representaciones Sociales

\begin{abstract}
This article proposes to reveal the social representations of citizenship and citizenship education by students in Pedagogy of History, Geography and Social, in two universities based in the Metropolitan Region of Chile, specifically in relation to their formal training as teachers and associated curriculum for their professional development. In this sense, the study adheres to a constructivist paradigm based on a qualitative approach. The design is linked to a case study and the data were collected using documentary analysis and a focus group discussion, the former which was analyzed via content analysis. The results of the study showed that students' social representations are linked to different citizenship paradigms, as well as two different types of citizenship formation. In addition, students' social representations comprise of a mixture of theoretical elements with respect to citizenship and citizen education. We observed, at the same, in these social representations of citizenship, characteristics of a communitarian and liberal paradigm, or one based on political and social formation.
\end{abstract}

Keywords: Curriculum, Teacher training, Citizenship, Social Representation.

Héctor Gómez Cuevas y Felipe Pizarro,

Representaciones Sociales y Formación Inicial Docente: El desafío de educar en ciudadanía, Autoctonía. Revista de Ciencias Sociales e Historia, Vol. I, N², Julio-Diciembre 2017, 284-299 ISSN 0719-8213 


\section{El Problema}

El desarrollo de la ciudadanía es una labor de la sociedad en su conjunto, tanto delEstado, las familias, las agrupaciones civiles y la escuela. No obstante, la escuela juega un rol mucho más protagónico en este proceso de formación, entregando conocimiento, experiencias, habilidades, actitudes, etc. que llevarán a los estudiantes a desarrollarse como ciudadanos en un mundo complejo y globalizado. En tal sentido, es importante reconocer que en 'este mundo globalizado' han cambiado muchos elementos constitutivos de la sociedad, entre ellos uno de los más impactantes para la labor educativa ha sido el acceso masivo de los sujetos a las instituciones educativas formales (Imbernón, 2005) y, junto con ello, una extensión de la 'carrera' educativa de los jóvenes. Además, la ampliación de la cobertura escolar -como cambio paradigmático de los últimos cuarenta años- ha dado paso a una mayor demanda de estudios superiores por parte de los estudiantes que egresan de la escuela, lo que se demuestra en un estudio realizado por el Programa de la Naciones Unidas para el Desarrollo (PNUD) donde la creación de universidades en Chile, junto con otros factores más, dieron paso a un aumento en las matrículas a dichas universidades, proceso que ha sido potenciado en los años noventa, y que se ha mantenido hasta la actualidad (PNUD, 2005).

Desde esta perspectiva, la universidad debe formar ciudadanamente a los estudiantes además de entregar conocimientos y habilidades específicas de cada carrera. De hecho, un estudio reciente de Martha Curcio y Edna Camargo (2012) sobre la relación entre universidad y formación ciudadana en Colombia, demostró que dicha formación "es una responsabilidad ineludible de la universidad" (124). En tal aspecto, el rol de la universidad no debe sólo centrarse en desarrollar habilidades que preparen para el mundo laboral a los sujetos, sino que además debe continuar con el proceso de formación ciudadana iniciado en la escuela, logrando desarrollar "ciudadanos activos, con todo lo que ello implica en cuanto a responsabilidad social y compromiso de aportar a otros en la generación de conciencia, en lo que respecta al ejercicio de la ciudadanía" (Curcio y Camargo, 2012: 125).

Si en la actualidad la universidad juega un rol importante en el desarrollo de la ciudadanía, la
Formación Inicial Docente (en adelante FID) asume una responsabilidad mucho mayor en este proceso, debido específicamente a que son los docentes quienes asumen la tarea de educar en ciudadanía y derechos humanos(Magendzo, 2000). En tal sentido, la FID se está enfrentando a una época de grandes y rápidos cambios a nivel mundial, donde existe un contexto de crisis de las instituciones sociales, de incertidumbres, de profundas transformaciones en lo económico, tecnológico y cultural, pero por sobre todo, en una época donde la labor educativa pareciera estar desorientada, donde carga con un exceso de responsabilidades y, además, es objeto de presiones frente a las exigencias de resultados inmediatos (Imbernón, 2005). De esta forma, la FID debe asumir un sinfín de desafíos, y dentro de éstos retos se encuentra la formación de los futuros ciudadanos.

En la actualidad la FID debe cumplir con formar un perfil de docente que se adecue a las demandas laborales, tecnológicas y sociales del mundo internacional. Por lo mismo, los docentes preparados y de calidad son aquellos que "se caracterizan por tener un conocimiento amplio, una sólida capacidad pedagógica y la disposición para guiar y sostener a los estudiantes, además de una comprensión del clima cultural y social de la educación" (Szilagyi y Szecsi, 2011 en: Pedraja, Araneda, Rodríguez y Rodríguez, 2012: 16). Desde esta perspectiva, el docente es un profesional y trabajador que debe recibir en la universidad las 'competencias' necesarias para el mundo complejo de hoy.

Ahora bien, a pesar de que las habilidades ciudadanas no se declaran de forma explícita en el perfil del docente, los estándares para los profesores de enseñanza media publicados el año 2012 rescatan la idea de un docente capaz de promover el desarrollo, tanto personal como social del estudiante en el aula, por lo tanto, por lo menos de forma implícita, los profesores deberían recibir una formación que los habilite para desarrollar la ciudadanía en las aulas. No obstante, la situación de la formación ciudadana en las escuelas tampoco es muy alentadora, de hecho, el Informe de la Comisión de Formación Ciudadana realizado el 2004, sostiene que a partir de la aplicación de un nuevo currículo de formación ciudadana iniciado a fines de los noventa, los docentes en su gran mayoría han demostrado 
dificultades para comprender dicho currículo y para aplicarlo en las escuelas (MINEDUC, 2004a). Por lo tanto, tenemos a un docente que entrega únicamente saberes conceptuales (contenidos) y un estudiante que memoriza dichos saberes (Muñoz y Torres, 2014).

Se dijo anteriormente que uno de los protagonistas más importantes en el proceso de formación ciudadana en las escuelas es el docente. Por lo mismo, si la FID no considera este tópico o posee dificultades en torno al desarrollo de la ciudadanía, es de esperar que los resultados en las escuelas - por lo menos en torno a la ciudadaníasean preocupantes. Por esta razón, se espera que las universidades consideren temáticas como la ciudadanía, el respeto a los derechos humanos, el medioambiente, la tolerancia, la participación política, etc. y los adecue al currículo de formación inicial de cada carrera, logrando generar espacios y experiencias de ciudadanía en la formación de los docentes. En tal aspecto, Pilar Muñoz (2013) frente al estudio de los derechos humanos en la FID, sostiene que la preparación de los docentes "conlleva un desafío aún más complejo y profundo, pues no solo incluye la transmisión de saberes y procedimientos, sino, además la reflexión sobre las disposiciones con que cada persona se aproxima a este tema y el deber de construir un discurso profesional capaz de superar el sentido común, los prejuicios y particularismos sin fundamento" (85).

Es posible argumentar por tanto que la FID especialmente- de profesores de Historia, Geografía y Ciencias Sociales en Chile es bastante deficitaria acerca de la temática ciudadana, dando como consecuencia "una falta de apropiación curricular sobre la formación ciudadana una vez comenzado el ejercicio profesional de la docencia" (Reyes et al., 2013: 221). En consecuencia, si la formación ciudadana no está presente durante el proceso de FID, existe una alta probabilidad de que los profesores reproduzcan tanto una escasa, parcial, o equívoca formación para la ciudadanía en sus estudiantes.

El Informe de la Comisión de Formación ciudadana (2004) plantea que existe una falta de apropiación por parte de los docentes del nuevo currículo insaturado con la reforma de fines de los noventa y, además, uno de los tópicos más complejos y menos entendidos por parte de los docentes es precisamente el de la formación ciudadana. Por lo mismo, el currículo de Historia, Geografía y Ciencias Sociales formulado desde las autoridades centrales de educación no se entrega intacto en el aula, sino que es reformulado por los docentes en la medida de sus conocimientos y experiencias. En otras palabras, existe una brecha entre el currículo formulado desde el Estado y lo implementado por los docentes de Historia, Geografía y Ciencias Sociales en torno a la formación ciudadana (Muñoz et al., 2013). Del mismo modo, algunos autores han planteado la idea de que las prácticas docentes se han modificado levemente respecto a lo que había antes de la reforma, pero aun así siguen siendo insuficientes para los propósitos planteados en ese momento (Cerda et al., 2004).

En síntesis, la formación ciudadana en las escuelas posee graves dificultades para ser abordada de acuerdo a los planteamientos ministeriales. Esa responsabilidad que en gran parte recae en el docente, específicamente el de Historia, Geografía y Ciencias Sociales, debe ser analizada no sólo en función de la didáctica, el diseño curricular o las estrategias evaluativas utilizadas por el docente en la escuela, sino también en base a las representaciones que tiene el profesor respecto a la formación ciudadana, que lo llevan a actuar de una forma y no de otra. Esas representaciones que se generan a partir de muchos factores -entre ellos la FIDson claves para una comprensión profunda de los dilemas pedagógicos actuales, entendiendo que no se conoce lo suficiente sobre las representaciones sociales de estudiantes de pedagogía en esta asignatura en torno a la ciudadanía y la formación ciudadana.

A partir de lo anterior surgen las siguientes interrogantes ¿Qué representaciones sociales tienen los estudiantes de pedagogía en Historia, Geografía y Ciencias Sociales en torno a la ciudadanía y la formación ciudadana? y ¿Qué relación tienen dichas representaciones sociales con el currículo de formación inicial?

De esta forma, el objetivo general que aborda este artículo es develar las representaciones sociales de estudiantes de la carrera de Historia, Geografía y Ciencias Sociales en torno a la ciudadanía y la formación ciudadana supone profundizar en 
las subjetividades que poseen los sujetos y los discursos que éstos enuncian a partir de la relación con sus pares, de los espacios de sociabilización universitarios, el impacto del currículo de la carrera, entre otros.

Adicionalmente, la presente investigación de desglosa en tres objetivos específicos: describir el currículo de Formación Inicial Docente de las carreras de Historia, Geografía y Ciencias Sociales de dos universidades de la Región Metropolitana, a través de los perfiles de egreso, los planes de estudio y los programas de las actividades curriculares en torno al concepto de ciudadanía y la formación ciudadana; conocer las representaciones sociales de los estudiantes de Pedagogía en Historia, Geografía y Ciencias Sociales de dos universidades de la Región Metropolitana frente al concepto ciudadanía y formación ciudadana; finalmente, establecer la relación existente entre el currículo de la Formación Inicial Docente de la carrera de Historia, Geografía y Ciencias Sociales de dos universidades de la Región Metropolitana y las representaciones sociales de sus estudiantes en torno al concepto de ciudadanía y formación ciudadana.2. 2. Lentes Epistemológicos

\subsection{Curriculum}

Cuando se pensaba que la perspectiva crítica había dicho la última palabra respecto al currículo, surgió una nueva mirada teórica reconocida como 'postcrítica'. Si bien, en muchos estudios se habla de una teoría post-crítica a secas, es más conveniente considerarla como 'perspectiva' debido a que aún no posee una epistemología completamente clara (Tadeu da Silva, 2001). Investigadores como Thomas Popkewitz o Cleo Cherryholmes han tomado ciertos elementos post-estructuralistas para analizar el currículo y sus estudios por ende han sido catalogados como post-críticos. Si bien, la intención no es hacer similitudes entre un enfoque teórico post-estructuralista y la perspectiva curricular post-crítica, es importante reconocer que ambos nacen en respuesta al estructuralismo europeo y las perspectivas críticas respectivamente. En otras palabras, se puede decir que lo postcrítico ha extendido el campo de análisis crítico, complementando las relaciones estructurales de poder (estructura económica, política y social) con elementos propios del análisis del discurso y de la producción de significados sociales. Esta nueva perspectiva, incorpora al análisis curricular conceptos como: incertidumbre, indeterminación y fluidez (Tadeu da Silva, 2001).

Cuando se mira el currículo bajo una perspectiva post-estructuralista, se hace hincapié en los significados de las cosas. Estos significados no son preexistentes, sino que son producciones sociales y culturales. En consecuencia, el conocimiento y el currículo se producen a partir de relaciones sociales de poder, donde existen 'verdades' que deben ser puestas en juicio, no para saber cuál es la verdad absoluta, sino para conocer y analizar el proceso con el cual aquellos elementos han sido considerados como 'verdades' (Tadeu da Silva, 2001).

La intención de posicionarse desde esta perspectiva es demostrar que la perspectiva post-crítica de este estudio es la más consistente frente a las representaciones sociales que poseen los sujetos frente a los componentes del currículo. En tal sentido, se entiende por currículo en este estudio, no sólo los aspectos culturales, las estrategias de enseñanza y métodos de evaluación determinados por los grupos hegemónicos, sino por sobre todo se entiende como un espacio simbólico donde diversas representaciones de los sujetos en torno a los fenómenos educativos chocan, se complementan, se reproducen y se transforman con el fin de dar sentido a sus experiencias.

Los elementos teóricos que aporta una mirada post-crítica del currículo a los estudios de las subjetividades y las representaciones de docentes $\mathrm{y}$ estudiantes, frente a la formación ciudadana y los procesos de cambio curricular del sistema educativo, orientan al autor ha decidir mirar las representaciones bajo una óptica donde las luchas discursivas asumen un rol fundamental.

\subsection{Ciudadanía}

La concepción de ciudadanía es bastante compleja y su definición depende en gran parte de la perspectiva teórica con la que se mire. De esta manera, la visión tradicionaldelaciudadanía "seentiendeenun sentido amplio, como una condición de quienes pertenecen a una comunidad, dicha condición implica igualdad de derechos y de deberes para los ciudadanos y denota participación" (Curcio y Camargo, 2012: 120). Esta visión moderna es funcional al proceso 
de fortalecimiento de los Estado-nación del siglo XIX, donde la comunidad de sujetos que habita un territorio determinado adquiere un estatus político denominado ciudadanía. Sin embargo, esta concepción tradicional de la ciudadanía ligada fundamentalmente a los derechos y deberes políticos- ha ido evolucionando en las últimas décadas con nuevas miradas de la ciudadanía.

Una teoría de la ciudadanía que está fuertemente arraigada en la sociedad actual es la liberal, entendida como aquella que privilegia los derechos individuales de los sujetos, la autodeterminación y la diversidad individual (Cerda et al., 2004). La ciudadanía liberal comprende la democracia como un sistema político donde el Estado de Derecho debe proteger las libertades individuales administrando y regulando a los ciudadanos.

La teoría o paradigma comunitarista critica fuertemente a la concepción liberal acusándola de "profesar un individualismo desarraigado, incapaz de ofrecer a los individuos ideales de la vida personal y comunitaria" (Cortina, 1997 en: Jiménez, Bravo y Ossandón, 2013: 208). Por lo tanto, en respuesta a esa concepción, los comunitaristas ofrecen una ciudadanía orientada a la asociatividad del sujeto, donde la política es una construcción colectiva y la democracia es participativa (Cerda et al., 2004). En esta perspectiva, el Estado no debe ser neutral, sino que debe poseer una identidad propia, consecuente con los valores de la ciudadanía.

Por lo tanto, existen dos paradigmas ciudadanos claramente definidos; el liberal y el comunitarista. Cada uno de estos está integrado por tres dimensiones ciudadanas, la civil, la política y la social, sin embargo, los énfasis que estos ponen en los elementos que componen estas dimensiones hacen que sean diferentes, incluso contrapuestos.

Los contextos donde surgen las diferentes dimensiones ciudadanas son en cierta medida bastante distintos a lo que sucede hoy en el siglo XXI. El nuevo siglo ha traído consigo un fenómeno de conectividad nunca antes experimentado por la especie humana, tanto a nivel económico como a nivel tele-comunicacional, político, migratorio, etc. Por lo mismo, los paradigmas ciudadanos junto con sus diferentes dimensiones han debido asumir - no sin algunas contradicciones- los nuevos desafíos del mundo globalizado.

Laciudadanía, porlotanto, esunacondicióndelsujeto que le otorga derechos legítimos de participación en la sociedad, pero, dicha participación está limitada a la teoría social imperante, donde el ciudadano puede contribuir de forma limitada y por ende reproducir el sistema social imperante, o también, puede participar en la construcción de una nueva sociedad transformando y eliminando el sistema social hegemónico. En otras palabras, se está hablando del proceso existente entre una participación posible y una participación real, donde la primera mantiene un ciudadano receptor pasivo de mensajes, mientras que la segunda implica un ciudadano empoderado capaz de producir sus propios mensajes y propuestas de solución a los conflictos de forma colectiva (Gutiérrez, 2011).

\subsection{Formación Ciudadana}

Los modelos de formación ciudadana descritos anteriormente pueden estar presentes en los currículos escolares nacionales como también en los universitarios. En tal sentido, se han definido dos posturas curriculares en torno a la formación ciudadana, específicamente conocidas como 'minimalista y maximalista' (Kerr, 2002 en: Reyes et al, 2013). El caso de la postura curricular minimalista ha sido definida como aquella basada en la formación ciudadana tradicional, donde el contenido está por sobre el desarrollo de habilidades y actitudes ciudadanas (Reyes, 2013). La postura minimalista curricular de formación ciudadana es profundamente instrumental y ligada a los derechos y deberes políticos, por lo tanto podría ligarse perfectamente con la racionalidad curricular técnica (Grundy, 1998).

Por otro lado, la postura curricular maximalista de la formación ciudadana supera la mirada tradicional de formación cívica y busca el "desarrollo de habilidades y predisposiciones que ayuden al estudiantado a insertarse como genuinos actores de la sociedad de la que forman parte" (Reyes, 2013: 224). En otras palabras, la postura curricular maximalista se vincula directamente con la 'formación ciudadana' en el más amplio sentido del concepto y no solamente con saberes cívicos. Si hubiese que relacionar la postura maximalista del currículo con una racionalidad curricular, la 
teoría práctica y algunos elementos de la teoría crítica serían las más cercanas a dicha postura (Grundy, 1998). En síntesis, la postura curricular de la formación ciudadana está ligada directamente con el modelo de formación de ciudadanía que se adopte, a saber: el modelo de formación de ciudadanía política estaría basado en una postura minimalista del currículo, con un claro enfoque técnico de la enseñanza y del aprendizaje. Por otro lado, los modelos de formación de una ciudadanía social y activa tendrían una postura maximalista de la formación ciudadana con un enfoque curricular práctico. Finalmente, el modelo de formación ciudadanía crítico evidentemente tiene elementos de la postura curricular maximalista, con un enfoque crítico de la enseñanza y el aprendizaje.

Enelpresente estudiosemiraránlas representaciones sociales de los estudiantes universitarios a partir del paradigma comunitarista de la ciudadanía y del modelo de formación ciudadana crítica, que considera la racionalidad curricular crítica y poscrítica como proveedora de elementos de análisis de significaciones y construcciones discursivas colectivas de los sujetos, a partir de la aceptación, la resistencia o transformación de discursos oficiales o hegemónicos.

\subsection{Las Representaciones Sociales}

Las representaciones sociales pueden definirse como conceptos, imágenes, explicaciones, nociones, etc., que se originan mediante la experiencia de los sujetos en la vida cotidiana, a través de interacciones interindividuales. Dichas representaciones podrían igualarse a los mitos y creencias de las antiguas sociedades (Moscovici, 1981 en: Denegri, Cabezas, Sepúlveda, del Valle, González y Miranda, 2010). Por lo mismo, las representaciones sociales pueden ser concebidas como la aprehensión e interpretación de la realidad cotidiana, donde aquello que es desconocido puede ser convertido en algo familiar. Además, las representaciones sociales se caracterizan por reflejar la relación dialéctica entre lo social y lo psicológico, donde la dimensión social alude al origen y construcción colectiva de las representaciones sociales a partir de la interacción con otros y la dimensión psicológica referente a la construcción del pensamiento cotidiano de la mente del individuo (Jodelet, 1984; Banchs, 1999; Prado y Krause, 2004; Moscovici, 1986 en: Mazzitelli y Aparicio, 2010).

Si bien, la representación social es parte de la relación entre lo social y lo psicológico, no se puede dejar de lado el aspecto contextual de las representaciones. De esta manera, Abric (2001) plantea que las representaciones están sujetas a los contextos existentes en la realidad.

Es importante destacar que las representaciones sociales implican una forma de interpretar el mundo existente, así como también una manera determinada de actuar sobre la realidad. Esto quiere decir que las representaciones sociales orientan el comportamiento del sujeto, designan su forma de involucrarse con lo cotidiano y finalmente ayudan a legitimar sus acciones (Prado y Krause, 2004). De acuerdo a Jodelet (1984) la representación social "designa una forma de conocimiento específico, el saber del sentido común, cuyos contenidos manifiestan la operación de procesos generativos y funcionales socialmente caracterizados" (474). De esta manera, las representaciones sociales son un conocimiento del sentido común que se produce a partir de la interacción entre lo individual y lo grupal bajo un contexto cultural, institucional, político, económico y comunicacional determinado, dando como resultado una manera particular de interpretar y actuar en el mundo.

En síntesis, las representaciones sociales como experiencia individual $y$ colectiva, como conocimiento de sentido común, como forma de interpretar y actuar en el mundo, etc., deben tener ciertas condiciones previas para su existencia. Dichas condiciones permitirán generar una representación mediante el proceso de objetivación y anclaje de un conocimiento complejo. La incorporación de ese conocimiento en la mente del individuo deberá acomodarse a otras ideas preexistentes hasta que finalmente se tenga una representación social. 


\section{Marco Metodológico}

\subsection{Enfoque}

El presente estudio adhiere al constructivismo como paradigma de la investigación educativa, considerando elementos ontológicos como la construcción de la realidad en un contexto determinado y elementos epistemológicos como el carácter subjetivo de la investigación y la construcción del conocimiento (Lincoln y Guba, 2000: 168 en: Sandín, 2003: 31). Por lo mismo, la investigación bajo este paradigma puede ayudar a comprender profunda y holísticamente los fenómenos y las manifestaciones sociales en un momento y espacio determinado (Rodríguez y Valldeoriola, 2009).

Desde esta perspectiva, y en relación con el paradigma de investigación, este estudio asume el enfoque metodológico cualitativo lo que implica que el investigador, bajo este paradigma, debe estudiar la "realidad en su contexto natural tal y como sucede, intentado sacar sentido de, o interpretar, los fenómenos de acuerdo con los significados que tienen para las personas implicadas (Rodríguez, Gil y García, 1999: 32). De esta forma, este estudio que intenta develar las representaciones sociales de estudiantes de pedagogía en Historia, Geografía y Ciencias Sociales toma los significados de éstos y los interpreta bajo su contexto natural sin la intervención y control de variables externas, lo que permite en definitiva un conocimiento de la realidad que intenta aproximarse lo más posible a la forma en la que la perciben los sujetos.

En síntesis, esta investigación estudia elementos complejos y difíciles de abordar como son las representaciones sociales de estudiantes de pedagogía en Historia, Geografía y Ciencias Sociales en torno al concepto de ciudadanía y formación ciudadana. Por lo mismo, el enfoque investigativo cualitativo es el más adecuado por su naturaleza multi metodológica, por su carácter interpretativo, constructivista y naturalista, además de su flexibilidad y búsqueda de comprensión de los fenómenos. En tal aspecto, "los métodos cualitativos se pueden usar para obtener detalles complejos de algunos fenómenos, tales como sentimientos, procesos de pensamiento y emociones, difíciles de extraer o de aprehender por métodos de investigación más convencionales" (Strauss y Corbin, 2002: 13). Las representaciones sociales son esos elementos complejos de abordar que necesitan de las herramientas metodológicas cualitativas para ser investigados.

Cabe señalar que la investigación sobre las representaciones sociales de estudiantes de pedagogía en Historia, Geografía y Ciencias Sociales en torno a la temática ciudadana es bastante escasa. De hecho, existe poca literatura en torno a las representaciones sociales y la formación inicial docente, lo que conlleva a clasificar la presente investigación como un estudio de tipo exploratorio.

\subsection{Diseño}

La investigación se enmarcó en un enfoque metodológico cualitativo con un diseño metodológico de estudio de caso. Esto quiere decir que no existe la intención de intervenir ni controlar el contexto donde se desenvuelven los sujetos estudiados (Hernández et al., 2010).

Por otro lado, cabe mencionar que la naturaleza del problema de investigación amerita una profundización y análisis exhaustivo de las representaciones sociales que poseen los estudiantes en torno a la temática ciudadana. Por lo mismo, como estrategia de diseño de investigación se ha optado por el estudio de casos, ya que este "implica un proceso de indagación que se caracteriza por el examen detallado, comprehensivo, sistemático y en profundidad del caso objeto de interés" (García Jiménez, 1991: 67 en: Rodríguez et al., 1999: 92).

Para el presente estudio, se optó por un tipo de estudio de casos colectivo o múltiple debido a que se investiga el fenómeno de las representaciones sociales en dos universidades de la Región Metropolitana, examinandodichas representaciones de los estudiantes de pedagogía en Historia, Geografía y Ciencias Sociales y su relación con el currículo de FID de cada universidad escogida. En este sentido, la opción de estudiar casos múltiples no se propone como foco central comparar los dos casos escogidos sino más bien busca dar evidencias más convincentes, tener una mirada mucho más global y en última instancia robustecer la información extraída. Esto, no implica que no se realicen ejercicios pequeños de comparación entre 
los casos a estudiar pero, como se mencionó más arriba, el foco central del estudio no será comparar los casos, sino entregar un análisis mucho más global respecto a la relación entre el currículo de FID y las representaciones de los estudiantes.

Por otro lado, el análisis de los datos entregados por los estudiantes no será la única fuente de información, ya que además se analizarán el currículo de las carreras de pedagogías de las diferentes universidades seleccionadas. Por lo mismo, el estudio de casos colectivos o múltiples asume como unidad de análisis el carácter inclusivo. Esto quiere decir que el objeto de estudio será analizado a partir de los discursos de los estudiantes y el currículo de FID, dando como resultado múltiples unidades de análisis.

En síntesis, el diseño de investigación es un estudio de casos colectivo o múltiple. Se analizará a partir de diferentes ópticas, por lo mismo conlleva la categoría de inclusivo y busca explorar y describir profundamente el fenómeno de las representaciones sociales.

\subsection{Unidades de Análisis}

La elección de los sujetos de este estudio no considera elementos alazar, sino que existe unaintencionalidad en la decisión de los sujetos que serán estudiados. Dicha decisión se orienta a partir de ciertos criterios selectivos impuestos por el investigador con el fin de conocer las representaciones que poseen los estudiantes de Pedagogía en Historia, Geografía y Ciencias Sociales sobre la ciudadanía y la formación ciudadana y su relación con el currículo de FID. Entre estos criterios, cabe mencionar los siguientes:

En primera instancia, para el primery tercer objetivo específico de este estudio se establece una muestra de documentos curriculares como; el perfil de egreso de la carrera de Pedagogía de Historia, Geografía y Ciencias Sociales de las dos universidades a estudiar, el plan de estudio o malla curricular y los programas de las actividades curriculares. La elección de los documentos obedece a su naturaleza declarativa y oficial, respecto a lo que sostiene la universidad como sus intenciones académicas. Por lo tanto, los tres documentos seleccionados tienen la característica de ser oficiales de las universidades, lo que implica que su estudio permite conocer el discurso oficial de dichas universidades, específicamente el discurso curricular de las carreras de Pedagogía en Historia, Geografía y Ciencias Sociales.

Por otro lado, respecto del segundo y tercer objetivo específico, se seleccionó una parte de los sujetos del universo, donde se establecen criterios para definir dicha muestra, entre los que se cuenta:

A nivel de institución de educación superior:

- Universidades que forman profesores de Historia, Geografía y Ciencias Sociales enseñanza media.

- Deben ser una universidad privada con sello laico y una universidad privada con sello confesional con el fin de poseer una visión más amplia sobre el fenómeno de las representaciones sociales. Como son dos universidades, pasarán a llamarse desde ahora caso 1 y caso 2.

- Deben ser de fácil accesibilidad con el propósito de no entorpecer los procedimientos investigativos.

A nivel de los estudiantes de pedagogía en Historia, Geografía y Ciencias Sociales:

- Estar cursando el último semestre de pedagogía en Historia, Geografía y Ciencias Sociales educación media. Estar cursando el último semestre implica que son estudiantes que prontamente estarán ejerciendo en los centros educativos, y por lo demás estar cursando el último semestre implica haber participado de la gran mayoría de las actividades curriculares que ofrece la carrera, lo que permite analizar las representaciones de los estudiantes a partir su experiencia académica casi completa.

- Haber realizado alguna experiencia de práctica profesional en una institución de educación media. Esto, debido a que gran parte de los estudios sobre formación ciudadana se han centrado en la educación básica, por lo que conocer y develar las representaciones de estudiantes de Historia, Geografía y Ciencias Sociales que han tenido 
alguna experiencia en educación media contribuye a ampliar el conocimiento sobre la formación ciudadana en las escuelas.

- Haber cursado y aprobado los cursos respectivos de ciudadanía y formación ciudadana declarados por el director y/o coordinador de dicha carrera.

En suma, se establecen los criterios para definir a los sujetos de la investigación, tanto en sus aspectos físicos como sociales (universidades y estudiantes). De esta forma, es posible obtener la información necesaria en pos del segundo y tercer objetivo específico. En suma, tanto los documentos curriculares seleccionados como los sujetos a investigar contribuyen a realizar el objetivo general de este estudio (Ver Cuadro 2).

\subsection{Técnicas e Instrumentos}

En primer lugar, se optó por la técnica de análisis documental entendida como el "análisis de contenido de los documentos, es decir, del mensaje informativo que contiene un documento plasmado en un texto, imagen, sonido, o multimedia" (Herrero, 1997: 44-45). De esta manera, la intención de esta técnica es tomar documentos de acceso público como de acceso interno con el fin de revisar su contenido a partir de los conceptos abordados en el marco teórico de esta investigación.

Parallevaracaboelanálisis documental, fuenecesario construir una matriz de análisis que ayudará en el proceso de revisión de los documentos curriculares. Dicha matriz establece los documentos que se mirarán y los criterios con los que se analizarán, organizándose en primer lugar a partir del perfil de egreso, donde se contemplan dos criterios de análisis: 1. La racionalidad curricular predominante y 2 . Si hace referencia explícitamente a la ciudadanía y/o formación ciudadana. En segundo lugar, respecto al plan de estudio o malla curricular, los criterios son los siguientes: 1 . Presenta actividades curriculares donde se aborde de forma explícita la ciudadanía y/o la formación ciudadana, 2. ¿Qué actividades curriculares? y 3 . Porcentaje total de actividades curriculares que abordan la temática de la ciudadanía y/o la formación ciudadana. Finalmente, en cuanto a los programas de estudio de las actividades curriculares, se establecieron como criterios: 1. Paradigma y/o dimensión ciudadana que promueve y 2. Tipo de formación ciudadana que promueve en los estudiantes.

En segunda instancia, se ha optado por la técnica del grupo focal o sesiones en profundidad definida como "una especie de entrevistas grupales, las cuales consisten en reuniones de grupos pequeños y medianos (tres a 10 personas), en las cuales los participantes conversan en torno a uno o varios temas en un ambiente relajado e informal, bajo la conducción de un especialista en dinámicas grupales (Hernández et al., 2010).

Por esta razón, esta técnica contribuyó a abordar el segundo objetivo específico referente a 'conocer las miradas de los estudiantes de Pedagogía en Historia, Geografía y Ciencias Sociales de distintas universidades de la Región Metropolitana frente al concepto ciudadanía y formación ciudadana'. Por otro lado, dio cumplimiento del tercer objetivo específico referente a 'establecer la relación existente entre el currículo de la Formación Inicial Docente de la carrera de Historia, Geografía y Ciencias Sociales de las dos universidades y las representaciones sociales de sus estudiantes en torno al concepto de ciudadanía y formación ciudadana'.

Para realizar el grupo focal se creó un guion de grupo focal orientado hacia las temáticas de ciudadanía y formación ciudadana, con una batería de preguntas destinadas a estudiantes de último semestre de la carrera de Pedagogía en Historia, Geografía y Ciencias Sociales. Dicho guion, está organizado en base a la dimensión transubjetiva de las representaciones sociales, entendida como aquella donde el participante se posiciona como estudiante de pedagogía y sus respuestas se guían a partir de sus experiencias vividas como estudiante universitario. En ese sentido, la dimensión transubjetiva del guion se dividió en cuatro categorías: 1. La ciudadanía como concepto teórico, 2. La formación ciudadana, 3. La relación entre currículo de Formación Inicial Docente (FID) y la ciudadanía, y finalmente, 4. La relación entre el currículo de FID y la formación ciudadana. 
H.Gómez y F. Pizarro, Representaciones Sociales y Formación Inicial Docente

Autoctonía. Revista de Ciencias Sociales e Historia, Vol. I, N²

Cuadro 2. Esquema resumen metodología de estudio

\begin{tabular}{|c|c|c|c|c|c|}
\hline OBJ. & TÉCNICA & INSTRUMENTO & $\begin{array}{l}\text { TIPO DE } \\
\text { ANALISIS }\end{array}$ & MUESTRA & $\begin{array}{l}\text { CRITERIOS DE } \\
\text { SELECCIÓN }\end{array}$ \\
\hline 1 & $\begin{array}{l}\text { Análisis } \\
\text { documental. }\end{array}$ & $\begin{array}{l}\text { Matriz de } \\
\text { análisis. }\end{array}$ & $\begin{array}{l}\text { Análisis de } \\
\text { contenido. }\end{array}$ & $\begin{array}{l}\text { Documentos } \\
\text { curriculares: Perfil } \\
\text { de egreso, plan de } \\
\text { estudio y programas } \\
\text { de estudio. }\end{array}$ & $\begin{array}{l}\text { Oficiales } \\
\text { Declarativos }\end{array}$ \\
\hline 2 & $\begin{array}{l}\text { Grupo } \\
\text { focal. }\end{array}$ & $\begin{array}{l}\text { Guion de grupo } \\
\text { focal. }\end{array}$ & $\begin{array}{l}\text { Análisis de } \\
\text { contenido }\end{array}$ & $\begin{array}{l}\text { Estudiantes de la } \\
\text { carrera de } \\
\text { Pedagogía en } \\
\text { Historia, Geografía y } \\
\text { Ciencias Sociales }\end{array}$ & $\begin{array}{l}\text { Señalados en el } \\
\text { texto }\end{array}$ \\
\hline 3 & $\begin{array}{l}\text { Análisis } \\
\text { documental } \\
\text { y grupo } \\
\text { focal. }\end{array}$ & $\begin{array}{l}\text { Matriz de } \\
\text { análisis y grupo } \\
\text { focal. }\end{array}$ & $\begin{array}{l}\text { Análisis de } \\
\text { contenido }\end{array}$ & $\begin{array}{l}\text { Documentos } \\
\text { curriculares y } \\
\text { estudiantes de } \\
\text { Pedagogía en } \\
\text { Historia, Geografía y } \\
\text { Ciencias Sociales. }\end{array}$ & $\begin{array}{l}\text { Señalados en el } \\
\text { texto }\end{array}$ \\
\hline
\end{tabular}

Fuente: Elaboración propia.

\section{Presentación de Resultados}

Los resultados de este estudio están basado en el análisis de dos universidades de la Región Metropolitana: El Caso 1 (en adelante CI) y el Caso 2 (en adelante C2). Ambas instituciones son de dependencia privada; el CI tiene un carácter confesional mientras que el C2 un carácter laico. En ambos casos se analizó la documentación y representaciones de estudiantes de la carrera de Pedagogía en Historia, Geografía y Ciencias Sociales.

Respecto de los hallazgos del primer objetivo específico, es posible mencionar que el currículo de FID delCl declarauna racionalidad curriculartécnica que se vincula con la promoción de una ciudadanía basada en el paradigma liberal con una dimensión civil y política. De este modo, el tipo de formación ciudadana que promueve este currículo se orienta hacia una formación política de los ciudadanos, limitando las posibilidades de los estudiantes a desarrollar habilidades de formación centradas en los nuevos desafíos del mundo actual (Cerda et al., 2004). En cuanto al C2, el currículo de FID posee elementos mucho más ligados a una racionalidad práctica, promocionando una ciudadanía basada en mayor medida en un paradigma liberal y en menor medida en un paradigma comunitarista. Frente a esto, pareciese que el currículo de FID del C2 posee un eclecticismo teórico, donde se muestran elementos liberales como comunitarista. No obstante, la orientación mayoritaria es hacia el liberalismo con la dimensión ciudadana civil y política. Además, el currículo rescata elementos culturales e identitarios que promueven una dimensión ciudadana cultural, elemento que está ausente en el Cl.

Por otro lado, el currículo de ambos casos muestra solo una actividad curricular destinada explícitamente a abordar la ciudadanía. En el Cl, se identificó 'Ciudadanía y vida cívica' donde el paradigma ciudadanos predominante es el liberal en base a sus dimensiones civil y político. Por otro lado, en el C2, se identificó la asignatura 'Fundamentos de las ciencias sociales II' donde al igual que en el Cl se promueve una ciudadanía liberal basada en la dimensión civil y política. Respecto al resto de los 
programas analizados, en los dos casos no aluden abiertamente a la ciudadanía pero implícitamente se pudo identificar ciertos elementos que permitieron hacer ciertas deducciones. En el Cl, las actividades curriculares revisadas aluden en su mayoría a un paradigma liberal y mínimamente se percibe la presencia de elementos comunitaristas. En el C2, el resto de las actividades curriculares aluden de forma implícita a la ciudadanía, donde gran parte de los elementos curriculares se orientan a un paradigma liberal, y unos pocos a una dimensión comunitarista. El aspectos rescatable del C2, es que -a diferencia del C1- promueve en gran parte una dimensión ciudadana cultural, elemento que le permite asumir con mayor autoridad los nuevos desafíos ciudadanos del siglo XXI (Reguillo, 2003).

Del mismo modo, en los dos casos analizados (C1 y C2) las actividades curriculares de 'Didáctica de la Historia' no presentan alusión explícita a entregar herramientas para formar ciudadanos en las escuelas. En este sentido, los cursos en ambos casos se orientan por entregar estrategias y métodos para enseñar adecuadamente la disciplina historiográfica, pero en ningún caso relacionada con la formación de ciudadanos. De esta manera, en ninguno de los currículos de los casos estudiados existe la presencia de una actividad curricular destinada a las ciencias sociales, fenómeno que aleja aún más las posibilidades de fortalecer este tema en el proceso de formación inicial. De esta forma, los dos casos estudiados se suman a la gran lista de universidades que no poseen cursos de didáctica especializados en ciencias sociales (Cox et al., 2014).

Por otra parte, se logró notar que gran parte de las actividades curriculares seleccionadas por las autoridades académicas del C1 y del C2 pertenecen a la disciplina historiográfica o geográfica. Por lo tanto, el espacio para el estudio de las ciencias sociales, específicamente los cursos destinados al trabajo de la ciudadanía, es escaso, lo que en alguna medida provoca que la temática ciudadana siga teniendo un papel secundario en la formación de los docentes, siendo parte de un curso de Historia o de un curso de Geografía.

Respecto del segundo objetivo específico es menester señalar en primera instancia que los estudiantes de ambos casos conciben la ciudadanía como el ser parte de una sociedad donde se participa dentro de un sistema social. Dicha participación se entiende de formas distinta según los casos, en cuanto al $\mathrm{Cl}$, los estudiantes manifestaron que la ciudadanía consistía en participar activamente dentro de la sociedad construyéndola colectivamente. Por otro lado, en el C2 los estudiantes manifestaron que la ciudadanía era una instancia de participación basada en los derechos, los deberes y la cultura. En consecuencia, se concluye que las miradas de ciudadanía son similares pero la concepción de participación discrepa entre uno y otro caso, donde el $\mathrm{Cl}$ asume elementos ligados al comunitarismo y el C2 elementos mucho más liberales.

En segundo lugar, respecto al balance de los ciudadanos de hoy, los dos casos mencionaron que existen ciudadanos que participan y los que no. Para el C1, los que participan son aquellos que se organizan en sectores locales como juntas de vecino o clubes deportivos. Y para el C2, los ciudadanos que participan hoy son aquellos autónomos y consientes del voto que ejercen. Las miradas que poseen los estudiantes respecto a los ciudadanos de hoy entran en sintonía con su concepción de ciudadanía, lo que produce, en consecuencia, que la mirada del Cl esté ligada a elementos comunitarista y para el C2 componentes del paradigma liberal. En torno a lo sostenido anteriormente es que se concluye que las dos miradas no son excluyentes entre si debido a que, desde la teoría, los elementos de ambos paradigmas ciudadanos no siempre entran en contradicción, sino que en muchos casos tiene que ver con el énfasis que le dan a cierto tipo de participación ciudadana, donde para el comunitarista la participación real radica en la decisión colectiva en sectores locales mientras que para el liberal la participación mediante derechos y deberes hace hincapié en el ejercicio del voto más que en otros ámbitos de participación.

En tercer lugar, se puede mencionar que existe un eclecticismo teórico o una mezcla de elementos teóricos de la ciudadanía dentro de las miradas que poseen los estudiantes en ambos casos. En el C1, los estudiantes manifestaron que el ciudadano ideal es aquel que es activo políticamente y participa mediante el voto, pero también mediante organizaciones locales. Por lo tanto, existe una mezcla de elementos liberales y comunitaristas que -se insiste-no necesariamente son excluyentes, sino que poseen énfasis distintos respecto de las 
prácticas ciudadanas. Para el C2, pasa exactamente el mismo fenómeno que el $\mathrm{Cl}$ donde los estudiantes tienen una visión del ciudadano ideal como aquel sujeto autónomo e independiente que es consciente de su voto. Esto permitió deducir que los estudiantes de Pedagogía poseen en sus visiones una mezcla de elementos liberales y comunitaristas que no son excluyentes entre sí. Esta mezcla de elementos teóricos, en las miradas de los estudiantes, puede deberse a los diferentes ámbitos de influencia en lo que se encuentran insertos como la familia, el barrio, la relación entre pares estudiantiles, los medios de comunicación, el currículo de la carrera que los forma como profesores, etc.

En cuarto lugar, respecto a la formación ciudadana, para ambos casos se concibe como un proceso donde se forma al individuo para que sea parte de la sociedad. Para el Cl, ese proceso está asociado a la cotidianeidad de los sujetos, es decir, que el individuo se forma ciudadanamente a partir de lo que ocurre en su diario vivir. Esto demuestra claramente una orientación hacia una formación ciudadana social. Para el C2, la formación ciudadana debe estar más ligada hacia el ámbito político o hacia una formación ciudadana más política, lo que entra en contradicción con su mirada sobre las prácticas ciudadanas más adecuadas para el mundo de hoy, donde aludieron a elementos de una dimensión más social que política. Se insiste, las contradicciones y mezclas de elementos teóricos de los estudiantes demuestra que existen diversas esferas de influencias que alimentan sus representaciones de la realidad.

En quinto lugar, los estudiantes de pedagogía de los dos casos estudiados perciben una orientación hacia una formación ciudadana política por parte del currículo de FID, elemento que se condice con lo declara de dicho currículo. En este sentido, se afirma que el currículo de FID de los dos casos estudiados mantiene una formación ciudadana ligada a elementos cívicos, lo que en otras palabras significa que no han tomado en cuenta las recomendaciones hechas por las comisiones ministeriales y la literatura especializada en torno al tema (MINEDUC, 2004a; Cox et al., 2014). Por lo demás, los estudiantes corroboran la mínima presencia de actividades curriculares destinadas a la ciudadanía y/o formación ciudadana en el currículo de FID de los dos casos. En el C1 se menciona 'Ciudadanía y vida cívica' y en el C2 sólo
'Fundamentos de las ciencias sociales II'. En este sentido, existe una bajísima presencia de la temática ciudadana lo que es preocupante, insistimos, para el perfeccionamiento de la formación de ciudadanos en la escuela. Si no existe el espacio suficiente en las universidades para desarrollar la ciudadanía los docentes en las escuelas seguirán manteniendo las mismas prácticas antiguas, en base a sus experiencias escolares.

Finalmente, los estudiantes del Cl mencionaron como ámbitos de formación ciudadana espacios extracurriculares, como iniciativas de ayuda social (ejemplificaron con el terremoto de febrero del 2010 y con el incendio de Valparaíso este año 2014) que se originaron dentro de la misma universidad. En este sentido, es posible notar dos cosas: la primera es que el currículo de FID del Cl no está proveyendo de los espacios necesario para desarrollar la ciudadanía en sus estudiantes y la segunda es que esta declaración de los estudiantes permite confirmar la tesis de que existe diversos espacios de formación ciudadana donde los sujetos alimentan sus miradas y concepciones sobre los fenómenos sociales, en este caso sobre la ciudadanía y la formación ciudadana.

Respecto al objetivo tres de esta investigación se puede mencionar en primer lugar que, a partir del estudio de las declaraciones curriculares oficiales de los casos estudiados, sólo se pudo establecer la relación existente entre representaciones y currículo de FID, pero no se logró determinar el impacto del currículo sobre las representaciones, tampoco el nivel de influencia, ni mucho menos si las representaciones sociales de los estudiantes han sido alimentadas por el currículo de FID. Esto, porque los datos que entregó el análisis documental no permitieron hacer otro tipo de aseveraciones más que determinar una relación.

En segundo lugar, respecto a la representación social de ciudadanía y lo declarado por el currículo de FID, el Cl posee encuentro y desencuentros. Respecto a los encuentros, se puede mencionar que aquellos elementos liberales que componen las representaciones de los estudiantes (en menor medida que el C2), específicamente los que aluden a una participación mediante el sufragio tienen una relación directa con el currículo de FID que promueve precisamente una ciudadanía liberal con una dimensión civil y política. 
Por otro lado, respecto a los desencuentros existentes entre las representaciones sociales y lo declarado por el currículo de FID, se pueden mencionar que la mayoría de los elementos que componen la representación social de ciudadanía que poseen los estudiantes aluden al comunitarismo basada preferentemente en una dimensión política y social de la ciudadanía, donde se destaca la organización local, barrial, la participación a partir de la construcción social y no sólo a partir de la adaptación pasiva y la acción colectiva por sobre la individual.

Por otra parte, el currículo de FID del C1 promueve una ciudadanía liberal basada en sus dimensiones civil y política, lo que demuestra que no existe relación entre la representación de los sujetos y lo declarado oficialmente por la carrera. Lo mismo sucede con la formación ciudadana, donde los estudiantes del Cl, en sintonía con su representación de ciudadanía, conciben la formación ciudadana como el proceso donde se forman los sujetos para participar en la sociedad y en la construcción de esta. Dicha formación se realiza mediante cotidianeidad de los sujetos, a partir de lo que ocurre en su entorno local y, desde ahí, se avanza hacia lo global hacia esferas institucionalizadas. En este sentido, la representación de la formación ciudadana que poseen los sujetos del Cl es de una formación social de la ciudadanía. No obstante, el currículo de FID promueve una formación ciudadana política, lo que demuestra nuevamente que no existe una relación entre la representación de los estudiantes y lo declarado por el currículo.

Es importante notar que las representaciones de los estudiantes del Cl sobre la ciudadanía demuestran un eclecticismo teórico, donde conviven elementos liberales y comunitaristas. Claro está, que los elementos que se entremezclan en dichas representaciones no son excluyentes entre sí, lo que de alguna manera no produce contradicciones en la forma de representar la ciudadanía por parte de los sujetos del Cl. Por otra parte, se afirma que gran parte de los componentes de las representaciones de los sujetos del Cl sobre ciudadanía y formación ciudadanía aluden al comunitarismo y la formación ciudadana social respectivamente. Por lo mismo, como no existe relación entre esta representación y el currículo de FID del C1, se deduce que existen otros espacios sociales distintos a los propuestos por el currículo de la carrera que alimentan dichas representaciones que las cuestionan y que las transforman. Dichos espacios podrían ser la familia, el barrio, los medios de comunicación, las relaciones entre pares estudiantiles, etc.

En tercer lugar, respecto a las representaciones sociales de los estudiantes del C2 y su vinculación con el currículo de FID, se concluyó que poseen mayoritariamente encuentros más que desencuentros. En cuanto a la ciudadanía, la representación social que poseen los sujetos alude a una inserción en la sociedad mediante los derechos, los deberes y la cultura. Dicha representación no aludió a una construcción colectiva de la sociedad, por lo que se dedujo que la representación de los estudiantes de pedagogía se basa en un paradigma liberal de la ciudadanía y en una dimensión civil, política y cultural. Por otro lado, el currículo de FID promueve precisamente un tipo de ciudadanía liberalbasada enla dimensión civil, políticay cultural. En consecuencia, existe una relación evidente entre la representación social de los estudiantes y lo que declara el currículo de FID. No obstante, algunos mínimos elementos de la representación social de ciudadanía que poseen los estudiantes del C2 aluden al comunitarismo, sobre todo aquellos ligados a la autogestión y organización a nivel micro, lo que no se condice con lo declarado por el currículo de FID. En tal sentido, se deduce primero una mezcla teórica en la representación social sobre ciudadanía $\mathrm{y}$, segundo, que existen otros ámbitos sociales que al parecer influyen en las representaciones sociales de los estudiantes respecto a la ciudadanía, dichos espacios podrían ser la familia, las organizaciones estudiantiles, los medios de comunicación, etc.

Respecto a la formación ciudadana, las representaciones sociales de los estudiantes del C2 sobre este tema aluden al proceso donde a los sujetos que se les inculca la concepción ciudadana, los valores ciudadanos, la concepción de derecho y de deber, etc. Dicha representación social está asociada a elementos de una formación ciudadana política, lo que se condice con el currículo de FID ya que este también promueve mayoritariamente una formación ciudadana de tipo política, lo que demuestra una evidente relación entre la representación de los sujetos del C2 y lo declarado por el currículo de FID. Incluso, para los sujetos del C2, las personas deben formarse ciudadanamente 
en base a una dimensión netamente política, lo que entra en sintonía con lo declarado por el currículo de FID.

En cuarto lugar, se puede mencionar que, entre los dos casos estudiados, el Cl es el que demuestra una menor relación entre las representaciones de los estudiantes de Pedagogía y lo declarado por el currículo de FID. Por otro lado, el C2 muestra una mayor relación entre las representaciones de los estudiantes y lo declarado por el currículo de FID. En este sentido, el C2 posee una relación más estrecha entre representación y currículo, lo que podría indicar hipotéticamente que el currículo de la carrera podría estar impactando mucho más en las representaciones de los estudiantes que en el C1, sin embargo, eso es solo una suposición ya que como se mencionó en párrafos anteriores los datos de esta investigación no permitieron hacer dicha aseveración.

Finalmente, se destaca que en los dos casos estudiados se visualiza una mezcla de elementos teóricos sobre la ciudadanía, tanto en las representaciones sociales de los estudiantes como en lo declarado por el currículo. Dichas mezclas demuestran que, para el caso de los estudiantes, existen diversos ámbitos de desenvolvimiento que podrían estar alimentando sus representaciones sociales y para el caso del currículo de FID puede existir una desorientación por parte de las autoridades académicas en cuanto al tema de la ciudadanía o, por otro lado, podría implicar una intencionalidad de mezclar elementos teóricos de la ciudadanía con la intención de entregar un bagaje mayor de herramientas conceptuales a los estudiantes. De acuerdo a lo analizado anteriormente se podría presumir que la primera opción se hace más evidente, donde el currículo de las carreras de los dos casos estudiados posee una desorientación que podría implicar un desconocimiento sobre el tema o también una falta de prioridad en torno a lo que implica el desarrollo de la ciudadanía en la formación de profesores.

\section{Conclusiones}

Los estudios sobre la formación ciudadana en Chile han tomado una fuerza significativa en los últimos años, sobre todo luego de que este tópico se instalara en el centro del debate educativo. Claramente el término de la dictadura militar contribuyó a la generación de políticas de recuperación de escenarios democráticos. En este sentido, este estudio se propone ser un aporte en dicha recuperación.

A partir del análisis realizado en el apartado anterior, es posible señalar que la FID sigue estando en deuda respecto a la formación de profesores capaces de desarrollar la ciudadanía en las escuelas. A nivel de diseño curricular, las oportunidades de formación en ciencias sociales, particularmente en torno a la ciudadanía siguen siendo escasas.

Por otro lado, es posible concluir que no existe una mirada univoca, sino por el contrario, existe una amplia diversidad de representaciones de la ciudadanía, particularmente sobre la participación en la sociedad. Los casos estudiados nos permitieron dar cuenta de que la representación social sobre participación ciudadana no se encasilla únicamente en el ejercicio del sufragio, sino también se concibe como una construcción de base, desde lo local y lo barrial.

Junto a lo anterior, se puede sostener que en los casos estudiados, el fenómeno que predomina a la hora de analizar las representaciones sociales sobre ciudadanía es el 'eclecticismo teórico'. No existe representación basada en aspectos únicamente liberales o únicamente comunitaristas, sino más bien una tendencia a la mixtura de ellos. En términos proyectivos, se podría suponer que los diseños curriculares de la FID que piensen en fortalecer la formación ciudadana deben tener en consideración este aspecto, sobre todo pensando que la prescripción en ámbitos ciudadanos implica necesariamente una intencionalidad valórica y por tanto ideológica de los sujetos que se pretende formar.

Por otro lado, cabe destacar que el 'eclecticismo teórico' señalado previamente está siendo alimentando por diversas esferas de influencia que impactan de manera directa en las representaciones de los sujetos. No es posible determinar qué esfera impacta más y cuál menos, pero si se puede afirmar que no existe una exclusiva. La FID es una más entre muchas.

Finalmente, el estudio permitió dar cuenta de la existencia de un ámbito científico que ha sido poco 
explorado por los expertos; dicho ámbito tiene relación con las representaciones de los sujetos universitarios en torno a la ciudadanía y con la influencia que podría tener el currículo de FID en dichas representaciones, entendiendo que los estudios han demostrado que el impacto de dicho currículo muchas veces es bastante débil, incluso nulo en las representaciones de los estudiantes. Si se considera que las representaciones sociales son un elemento que guía las prácticas docentes, se podría presumir que los estudiantes universitarios entran a formarse como docentes con ciertas concepciones de los fenómenos sociales y egresan de su proceso formativo con dichas concepciones inalteradas (Comisión de Formación Inicial Docente, 2005) y, por lo mismo, su quehacer en la escuela se basa en su propio proceso de socialización en la cultura. No obstante, la FID podría constituirse como un espacio de reflexión profunda y problematizadora de esas experiencias previas con el fin de ofrecer miradas alternativas que apunten a una efectiva promoción del pluralismo, la diversidad, los derechos humanos y todo aquello que contribuya a mejores convivencias.

\section{Bibliografía}

Abric, J. (2001): Prácticas sociales y representaciones. Filosofía y Cultura Contemporánea. México, Ediciones Coyoacán.

Banchs, M. (1999): "Representaciones sociales, memoria social e identidad de género", Akademos, 2 (1), pp. 59-76.

Cerda, A., L. Egaña, A. Magendzo, E. Santa Cruz y R. Varas (2004): El complejo camino de la formación ciudadana: Una mirada a las prácticas ciudadanas, Santiago, LOM Ediciones.

Chile. MINEDUC, (2001): Estándares de desempeño para la formación inicial docente. Santiago.

Chile. MINEDUC, (2004a): Informe Comisión Formación ciudadana. Santiago.

Chile. MINEDUC, (2004b): Formación ciudadana en el currículum de la reforma. Santiago.

Chile. MINEDUC, (2007): Marco para la buena enseñanza. Santiago.
Chile. MINEDUC, (2009): Objetivos Fundamentales y Contenidos Mínimos Obligatorios de la Educación Básica y Media, actualización 2009. Santiago.

Chile. MINEDUC, (2010): Estudio internacional de Educación Cívica y formación ciudadana ICCS 2009.

Chile. MINEDUC, (2012): Estándares orientados para carreras de Pedagogía en Educación Media: Estándares pedagógicos y disciplinarios. Santiago.

Chile. MINEDUC, (2013): Bases Curriculares $7^{\circ}$ a $2^{\circ}$ medio. Santiago.

Cox, C., C. García, y R. Mardones, (2014): Currículos comparados, percepciones docentes y formación de profesores para la formación ciudadana: Tendencias y proposiciones de Mejoramiento, Informe final.

Curcio, M. y E. Camargo (2012): "Universidad y formación ciudadana”, Reflexión Política, 14 (28), pp. 119-125.

Da Silva, T. (2001): Espacios de Identidad, Barcelona, Octaedro.

Denegri, M., D. Cabezas, J. Sepúlveda, C. Del Valle, Y. González y H. Miranda (2010): "Representaciones sociales sobre pobreza en estudiantes universitarios chilenos", Liberabit, 16 (2), pp. 161-170.

García-Huidobro, J. (2011): "La Política docente hoy y la formación de profesores”, Docencia, 16, pp. 12-22.

García, F. y N. de Alba (2012): La educación para la participación ciudadana entre dos polos: el simulacro escolar y el compromiso social, En Nicolás de Alba, Francisco García y Antoni Santisteban (Eds.), Educar para la participación ciudadana en la enseñanza de las ciencias sociales, Volumen I, Asociación Universitaria de Profesorado de Didáctica de las Ciencias Sociales, Sevilla, Díada Editora, pp. 297-306.

Grundy, S. (1998): Producto o praxis del curriculum. Madrid, Morata.

Gutiérrez, S. (2008): "El estudio de la ciudadanía desde una perspectiva sociocultural", Anuario de Investigación UAM, 10, pp. 88-14

Gutiérrez, S. (2011): "Representaciones sociales y construcción de la ciudadanía en jóvenes universitarios", Sinéctica, 1, pp. 1-19.

Hernández, R., C. Fernández y P. Baptista (2010): Metodología de la investigación. México, McGraw-Hill. 
Herrero, C. (1997): "La investigación en análisis documental”, Educación y Biblioteca, 83, pp. 44-46.

Imbernón, F. (2006): La profesión docente en la globalización y la sociedad del conocimiento. En Alberto Gómez y Juan Escudero (Coord.), La formación del profesorado y la mejora de la educación: políticas y prácticas, España, Editorial Octaedro, pp. 231-244.

Jiménez, M., L. Bravo y L. Ossandón (2013): La construcción de la ciudadanía contemporánea: nuevas posibilidades para la enseñanza de la historia. En Ignacio Muñoz y Luis Ossandón (Com.), La didáctica de la historia y la formación de ciudadanos en el mundo, Santiago, DIBAM, pp. 203-224.

Jodelet, D. (1984): La representación social: fenómenos, concepto y teoría. En S. Moscovici (comp.), Psicología Social II, Barcelona, Paidós, pp. 469-494.

Jodelet, D. (2008): "El movimiento de retorno al sujeto y el enfoque de las representaciones sociales", Connexion, 89 (1), pp. 25-46.

Lincoln, Y. y E. Guba (1985). Naturalistic Inquiry, California, Sage Publications.

Magendzo, A. (2000): "Educar en derechos humanos: $\mathrm{Si}$ no los educadores ¿quién? Si no ahora ¿cuándo?", Docencia, 12, pp. 71-81.

Martínez, M. (2006): "Formación para la ciudadanía y educación superior", Revista Iberoamericana de educación, 42 (1), pp. 85-102

Mazzitelli, C. y M. Aparicio (2010): "El abordaje del conocimiento cotidiano desde la teoría de las representaciones sociales", Eureka, 17 (3), pp. 636-652.

Mazzitelli, C., S. Aguilar, A. Guiaro y A. Olivera (2009): "Representaciones sociales de los profesores sobre la docencia: contenido y estructura", Educación, Lenguaje y Sociedad, 7 (6), pp. 265-290.

Muñoz, C., N. Vásquez y M. Sánchez (2013): “Percepciones del estudiantado sobre la democracia y los derechos humanos al finalizar la educación general básica: Un estudio desde las aulas de Historia”, Psicoperspectivas, 12 (1), pp. 95-115.

Muñoz, C. y B. Torres (2014): "La formación ciudadana en la escuela: Problemas y desafíos”, Revista electrónica Educare, 18 (2), pp. 233-245.

PNUD (2005): “Expansión de la educación superior en Chile: Hacia un nuevo enfoque de la equidad y la calidad. Temas de desarrollo humano sustentable", $\mathrm{N}^{\circ} 10$, Santiago, Ministerio de Educación.

Popkewitz, T. (1994): "Política, Conocimiento y Poder: Algunas cuestiones para el estudio de las reformas educativas", Revista de Educación, 305, pp. 1-29.

Prado, M. y M. Krause (2004): "Representaciones sociales de los chilenos acerca del 11 de septiembre de 1973 y su relación con la convivencia cotidiana y con la identidad chilena”, PSYKHE, 13 (2), pp. 57-72.

Reguillo, R. (2003): "Ciudadanía cultural. Una categoría para pensar en los jóvenes”. Revista del ITESO, 55 (1), pp. 27-37.

Reyes, L., J. Campos, M. Ossandón y C. Muñoz (2013): "El profesorado y su rol en la formación de los nuevos ciudadanos: Desfases entre las compresiones, las actuaciones y las expectativas", Estudios Pedagógicos, 34 (1), pp. 217-237.

Rodríguez, D. y J. Valldeoriola, (2009): Metodología de la investigación, México, FUOC.

Rodríguez, G., J. Gil, y E. García (1999): Metodología de la investigación cualitativa. Granada, Ediciones Aljibe.

Sandín Esteban, M. P. (2003): Investigación cualitativa en educación: Fundamentos y tradiciones. Madrid, McGraw Hill, Interamericana de España.

Strauss, A. y J. Corbin (2002): Bases de la investigación cualitativa: Técnicas y procedimientos para desarrollar la teoría fundamentada, Bogotá, Editorial CONTUS, Universidad de Antioquia.

Recibido: 30 de abril de 2017. Aceptado: 15 de julio de 2017. 